\title{
Information About Sunscreen on YouTube and Considerations for Sun Safety Promotion: Content Analysis
}

Anne K Julian ${ }^{1}$, MA, PhD; Jessica Welch ${ }^{1}$, BS; Maddison M Bean ${ }^{2}$, MS; Sarah Shahid ${ }^{1}$, BS; Frank M Perna ${ }^{1}$, EdD, $\mathrm{PhD}$

${ }_{1}^{1}$ Behavioral Research Program, Division of Cancer Control and Population Sciences, National Cancer Institute, Rockville, MD, United States

${ }^{2}$ College of Public Health and Human Sciences, Oregon State University, Corvallis, OR, United States

\section{Corresponding Author:}

Anne K Julian, MA, PhD

Behavioral Research Program

Division of Cancer Control and Population Sciences

National Cancer Institute

9609 Medical Center Dr

Rockville, MD, 20877

United States

Phone: 12402766175

Email: anne.julian@nih.gov

\section{Abstract}

Background: Sunscreen use is a popular sun protection method; however, application of sunscreen rarely meets the standards recommended for effectiveness. Access to information about how to effectively use sunscreen may play a role in proper sunscreen application. The internet is a common health information source; however, the quality of sunscreen-related content varies.

Objective: The objective of this study was to examine information about sunscreen in YouTube videos by video source.

Methods: In November 2017, the authors identified the 20 most popular YouTube videos (sorted by view count and relevance) for each of these 5 search terms: sunscreen cancer, sunscreen health, sunscreen information, sunscreen ingredients, and sunscreen natural. The inclusion criteria were English language and view count $>1000(\mathrm{~N}=111$ unique videos). We double-coded videos for standard recommendations for sunscreen use (eg, apply 20 minutes before sun exposure), use of outdated terminology, and recommendation of complementary sun safety strategies.

Results: The view counts ranged from 1100 to 671,142 (median 17,774, SD 109,651) and the average daily views ranged from 1 to 1448 (median 23, SD 234). End users $(46 / 111,41.4 \%$ ) and health care providers $(24 / 111,21.6 \%)$ were the most common sources, and none of the most popular videos were produced by federal agencies or cancer-related nongovernmental organizations. Health care provider videos included marginally more recommendations than end user videos (mean 1.46, SD 1.96 vs mean 1.05, SD 1.20), but few $(19 / 111,17.1 \%)$ mentioned reapplication. The videos were generally positive toward sunscreen $(82 / 111$, $73.9 \%)$; however, some videos were negative $(29 / 111,26.1 \%)$, with warnings about the health risks of chemical sunscreens and their ingredients. Do-it-yourself sunscreen tutorials represented 19/111 (17.1\%) of the sample.

Conclusions: YouTube is a potential source for disseminating sun safety messages; however, the quality of its sunscreen content varies. Most of the videos in our study failed to include important sunscreen use recommendations. Clinicians should be prepared to address the information needs of patients by discussing effective, evidence-based sunscreen application and recommending a combined sun safety approach.

(JMIR Dermatol 2020;3(1):e14411) doi: 10.2196/14411

\section{KEYWORDS}

sunscreen; social media; sun safety; cancer prevention

\section{Introduction}

Skin cancer affects an estimated 5 million people annually in the United States, with a treatment cost of over $\$ 8.1$ billion [1].
Ultraviolet (UV) radiation is a known carcinogen that causes direct and indirect DNA damage; intentional or unintentional UV exposure is responsible for the majority of skin cancer incidence [2]. Sunscreen is a primary sun protection strategy 
that is used by approximately one third of US adults [3]; however, sunscreen application rarely reflects the standards necessary to meet the advertised protection levels $[4,5]$. Furthermore, sunscreen is intended for use in combination with other sun protection strategies [6]. In 2012, the US Food and Drug Administration (FDA) issued new regulations for sunscreen labeling [7]; however, research has demonstrated that sunscreen application rarely reflects the thickness, evenness, and timing (of both initial application and reapplication) necessary to meet the advertised protection level of the sunscreen [8]. In 2014, the Surgeon General's Call to Action to Prevent Skin Cancer included a renewed call to promote a combination sun protection strategy (eg, wearing sunscreen, a hat, and protective clothing) [5].

The Centers for Disease Control and Prevention (CDC) and the American Academy of Dermatology support the daily use of sunscreen with a sun protection factor (SPF) of at least 15 and 30 , respectively, for UV protection, and dermatologists report recommending the use of sunscreen to their patients [9]. Although health care providers are trusted sources of health information, individuals increasingly supplement these sources with information garnered from the internet and social media [10]. In 2012, $72 \%$ of US adults had sought health information on the internet within the past 12 months [10]. The growing influence of social media in health decision making is not equally distributed across demographic and age groups; young age is a consistent predictor of social media use [11]; however, social media usage among adults is steadily increasing [12,13]. YouTube, the most prominent user-generated video-sharing website, allows any person to upload content on any topic [10]; based on traffic, it was the top-ranked website on the internet as of 2019 [14]. Furthermore, YouTube is increasingly being used as a platform to disseminate health information [15]. The American Cancer Society, National Institutes of Health, and National Cancer Institute have adopted this outlet to communicate health messages to broad audiences.

Despite these efforts, misleading or incorrect health information is common on YouTube [15]. For example, over one half of videos on the topic of immunization feature negative views or contradict the reference standard, which may lead viewers to make unsafe health decisions [16]. With respect to skin cancer, Basch et al [17] found YouTube content promoting untested home remedies for skin cancer, such as black salve; this finding highlights the challenge of health communication on social media. Moreover, a study of sun safety topics on YouTube [18] found that $17 \%$ of videos retrieved using the search term "sunscreen" included false or misleading information; however, less than a quarter of the videos in their sample were English language videos. Sunscreen use in practice is less thorough than recommended, and the question remains whether this is due in part to incomplete or misleading information available on social media platforms such as YouTube; this is especially likely to be the case if the content is not derived from governmental or professional medical organizations who create sun safety recommendations. Here, we analyzed a large sample of English language YouTube videos about sunscreen to address the following research questions: What primary sources, content types, positive/negative product attributions (valence), and adherence to standard recommendations [7] are included in YouTube videos about sunscreen? Do these videos contain the terms "sunblock" and "waterproof," which the FDA prohibits on sunscreen packaging? What complementary sun protection strategies do these videos include?

These data may inform public health video messaging to improve sun safety practices for effective sunburn prevention.

\section{Methods}

We conducted direct YouTube searches between November 13 and November 15, 2017 (search terms: sunscreen cancer, sunscreen health, sunscreen information, sunscreen ingredients, and sunscreen natural), deleting cookies after each search to avoid influencing subsequent results [19]. The YouTube region was set to United States. We identified the 20 most frequently appearing videos for each search term and removed duplicates to obtain the analytic sample of videos (see Figure 1).

We developed an early draft codebook in consultation with sun safety and communication experts to reflect common metrics in YouTube-based health information studies and sunscreen recommendation reference standards. This draft was then expanded and refined to include the recommendation of nonsunscreen sun safety methods, use of the outdated terms "sunblock" and "waterproof," and possible themes related to sunscreen safety. The codebook was pilot-tested using a test set of 5 videos by a team that included 3 experts in sun safety and 1 postbaccalaureate fellow. These videos were chosen using an extreme cases approach and reflected a diverse range of content and positions toward sunscreen. The codebook development team met to discuss and resolve coding discrepancies, generate additional constructs and codes, and refine coding response options.

After the initial codebook refinement, the coding team (MG, $\mathrm{SS}, \mathrm{JW}$, and AJ) double-coded all videos for the presence of 6 standard recommendations for effective sunscreen use based on FDA and CDC recommendations: SPF level, broad spectrum classification, application timing (20 minutes before exposure), liberal/generous application, and reapplication (every 2 hours and separately after toweling). We coded the overall valence toward sunscreen as negative or positive. We coded the use of the terms "sunblock" and "waterproof"; since 2011, the FDA has prohibited these terms on sunscreen labels, as they may be misleading [7]. We further coded the video source based on the posting source of the video and the posting channel's thumbnail and description. Lastly, we coded recommendation of the following complementary sun protection strategies (operationalized as instruction to adopt): seeking shade, wearing a hat, wearing protective clothing, wearing sunglasses, and staying indoors during peak UV hours (Cohen kappa range 0.80-0.96). Viewer attention to social media content is short [20]; therefore, we stopped coding each video after 5 minutes. Any final disagreement was resolved through discussion. We used Stata/SE 14 [21] to calculate descriptive statistics (means, medians and standard deviations, ranges and percentages) overall and stratified by video source. 
Figure 1. YouTube video search flow chart depicting the number of unique videos retrieved from YouTube in November 2017 using the search terms sunscreen information, sunscreen health, sunscreen natural, sunscreen ingredients, and sunscreen cancer and the final number of videos meeting the English language requirement. Relevance is a proprietary search algorithm owned by YouTube.

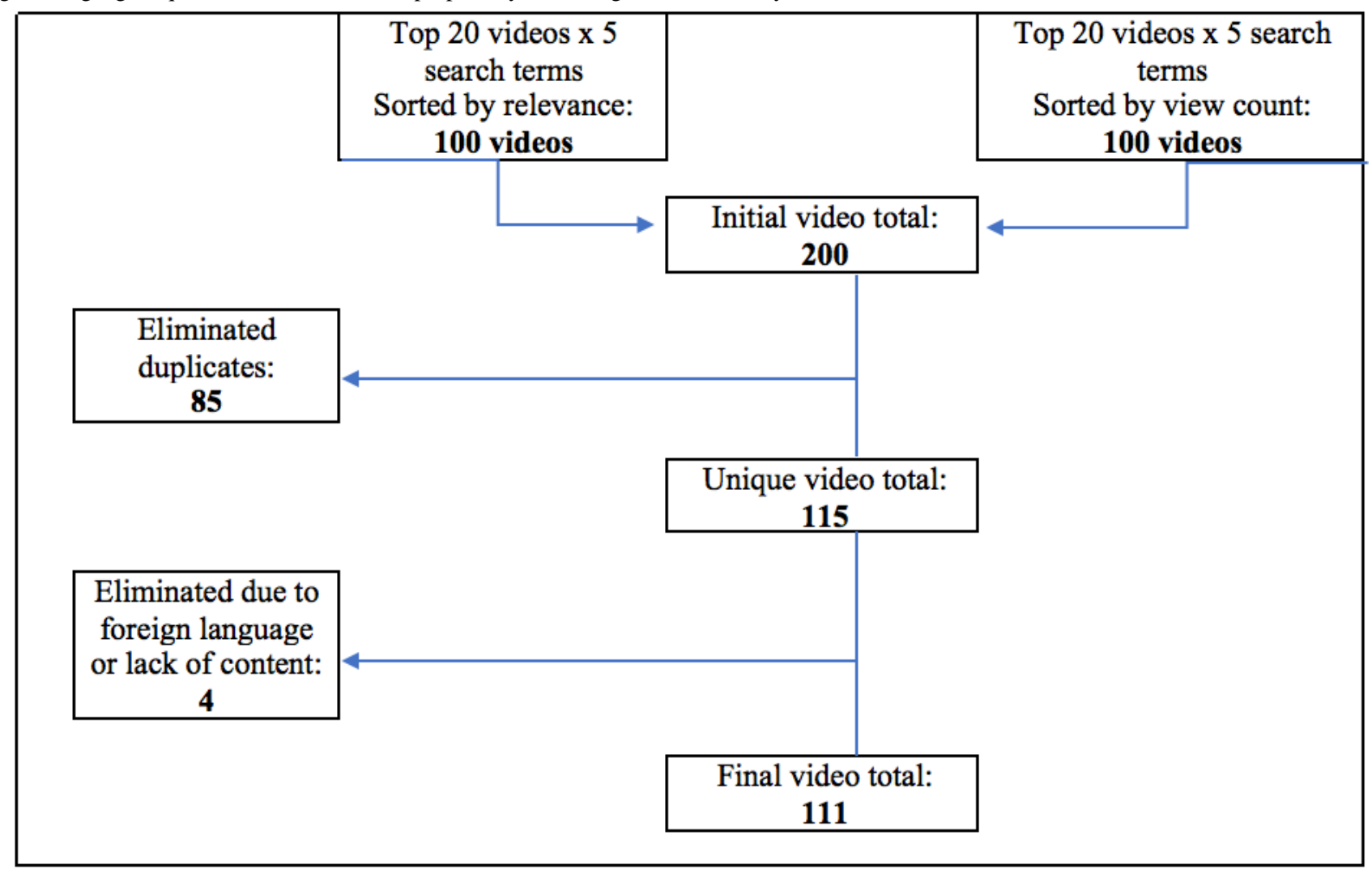

\section{Results}

The videos had view counts of 1100 to 671,142 (mean 58,642, median 17,774, SD 109,651), were between 40 seconds and 41 minutes long (mean 5:54 minutes, median 4:19 minutes), and were uploaded between 2009 and 2017 (average post duration 1127 days, median 1101, range 64-3135, SD 784). Average daily views ranged from 1 to 1448 (mean 96.8, median 23, SD 234). Primary video sources were health care providers or their associations $(24 / 111,21.6 \%)$, end users $(51 / 111,45.9 \%)$, and corporate entities $(15 / 111,13.5 \%$ ) (see Table 1 for the descriptive characteristics of the videos). No government health agencies were among the sources of videos retrieved. Valence toward sunscreen use was generally positive $(82 / 111,73.9 \%)$; however, positive content included promotion of mineral sunscreens that warned about health risks of chemical sunscreens. Videos that discouraged sunscreen use (29/111, $26.2 \%$ ) included misleading content, such as overstatement of the benefits of vitamin D, and outright falsehoods, such as the claim that sunscreen causes cancer.

Of the 6 recommendations for effective sunscreen use, the most frequently mentioned were SPF $(35 / 111,31.5 \%)$ and broad-spectrum classification $(30 / 111,27.0 \%)$; meanwhile, application timing, generous application, reapplication every 2 hours, and reapplication after toweling were each mentioned in fewer than $20 \%$ of videos (see Table 2). Most videos included 0,1 , or 2 recommendations. SPF was mentioned in 5/9 (55\%) of news and television coverage videos. Corporate entities, such as sunscreen or skin care companies, most frequently recommended generous application (4/14 videos, 29\%); however, $6 / 14(43 \%)$ of these videos paired this message with a poor demonstration of sunscreen application.

Few videos advocated the use of complementary sun protection strategies in conjunction with sunscreen $(19 / 111,17.1 \%)$. Of videos with positive valence toward sunscreen, $15 / 82(18.3 \%)$ suggested complementary sun protection strategies, compared to $3 / 29(10.3 \%)$ of videos with negative valence (see Table 2 ).

Few videos cited scientific sources for the presented information $(30 / 111,27.0 \%)$. Sources of scientific information were the Environmental Working Group (EWG), an environmental advocacy group $(13 / 111,11.7 \%)$, the FDA $(7 / 111,6.3 \%)$, and peer-reviewed articles (5/111 videos, 4.5\%). Lastly, an unexpected finding was that $19 / 111(17.1 \%)$ of videos demonstrated do-it-yourself recipes for how to make sunscreen at home. 
Table 1. Video content types, sources, and characteristics $(\mathrm{N}=111)$.

\begin{tabular}{|c|c|}
\hline Video characteristic & Frequency, n (\%) \\
\hline \multicolumn{2}{|l|}{ Content type } \\
\hline Public service announcement & $4(3.6)$ \\
\hline Medical advice & $24(21.5)$ \\
\hline Scientific explanation & $12(10.8)$ \\
\hline Product demonstration/promotion & $14(12.6)$ \\
\hline Do-it-yourself sunscreen tutorial & $19(17.1)$ \\
\hline Opinion/commentary & $23(20.7)$ \\
\hline Televised news clip & $15(13.5)$ \\
\hline \multicolumn{2}{|l|}{ Source } \\
\hline Nongovernmental organization & $5(4.5)$ \\
\hline News organization & $9(8.1)$ \\
\hline Health care provider ${ }^{\mathrm{a}}$ & $24(21.6)$ \\
\hline End user & $51(45.9)$ \\
\hline Corporation & $15(13.5)$ \\
\hline Topic-based group channel & $7(6.3)$ \\
\hline \multicolumn{2}{|l|}{ Use of outdated terms $\mathbf{b}^{\mathbf{b}}$} \\
\hline Sunblock & $13(11.7)$ \\
\hline Waterproof & $5(4.5)$ \\
\hline \multicolumn{2}{|l|}{ Valence $^{\mathrm{c}}$} \\
\hline Positive & $82(73.9)$ \\
\hline Negative & $29(26.1)$ \\
\hline \multicolumn{2}{|l|}{ Total number of recommendations } \\
\hline 0 & $54(48.6)$ \\
\hline 1 & $20(18.0)$ \\
\hline 2 & $19(17.1)$ \\
\hline 3 & $9(8.1)$ \\
\hline 4 & $3(2.7)$ \\
\hline 5 & $4(3.6)$ \\
\hline 6 & $2(1.8)$ \\
\hline
\end{tabular}

a“"Health care provider" was broadly defined to include physicians, nurses, and their organizations as well as people who self-identified as doctors but whose credentials did not include a medical degree. Videos featuring a doctor as the primary messenger were coded for the credentials of the provider; these included allopathic doctors (MD, DO), nonmedical doctors (PhD), and complementary or alternative medicine practitioners (DC, ND, MD(H)).

b In videos using the terms "sunblock" and "waterproof," 5 and 2 respectively used it in the context of an explanation that the term is considered misleading and should no longer be used.

${ }^{\mathrm{c}}$ Videos coded as positive toward sunscreen include those that promoted the use or home manufacture of mineral or "natural" sunscreens but that also included warnings about potential negative effects of chemical or commercial sunscreens on humans or the environment. 
Table 2. Standard recommendations for sunscreen use, overall and by video source ${ }^{\mathrm{a}}$.

\begin{tabular}{|c|c|c|c|c|c|}
\hline Recommendation & Overall $(\mathrm{N}=111)$ & $\begin{array}{l}\text { Health care } \\
\text { provider }(\mathrm{n}=24)\end{array}$ & $\begin{array}{l}\text { Corporation } \\
(\mathrm{n}=14)\end{array}$ & News $(n=9)$ & $\begin{array}{l}\text { End user } \\
(\mathrm{n}=47)\end{array}$ \\
\hline \multicolumn{6}{|l|}{ Sunscreen application, $\mathbf{n}(\%)$} \\
\hline Choose a broad spectrum & $30(27)$ & $5(22)$ & $2(14)$ & $4(44)$ & $14(30)$ \\
\hline SPF level 15+ & $35(32)$ & $8(36)$ & $3(21)$ & $5(55)$ & $16(37)$ \\
\hline Apply 20 minutes before exposure & $14(13)$ & $6(26)$ & $2(14)$ & $1(11)$ & $2(3)$ \\
\hline Reapply every 2 hours & $16(14)$ & $5(22)$ & $2(14)$ & $1(11)$ & $2(3)$ \\
\hline Apply a generous amount & $19(17)$ & $5(22)$ & $4(29)$ & $1(11)$ & $5(10)$ \\
\hline Reapply after toweling & $12(11)$ & $4(17)$ & $2(14)$ & $1(11)$ & $2(3)$ \\
\hline \multicolumn{6}{|l|}{ Complementary sun protection, $\mathrm{n}(\%)$} \\
\hline Wear a wide-brimmed hat & $1(4)$ & $1(4)$ & $1(0)$ & $2(22)$ & $2(4)$ \\
\hline Wear protective clothing & $12(11)$ & $1(4)$ & $0(0)$ & $3(33)$ & $3(6)$ \\
\hline Wear sunglasses & $8(7)$ & $1(4)$ & $0(0)$ & $2(22)$ & $1(2)$ \\
\hline Seek shade/use an umbrella & $11(10)$ & $0(0)$ & $0(0)$ & $3(33)$ & $3(6)$ \\
\hline Stay indoors during peak UV periods & $6(5)$ & $0(0)$ & $0(0)$ & $1(11)$ & $1(2)$ \\
\hline
\end{tabular}

a Due to small cell sizes, videos from nongovernmental organizations and sources coded as "Other," which included topic-based group channels and sources that were not classifiable, were omitted from these group analyses. Therefore, the rows do not total $100 \%$.

\section{Discussion}

\section{Principal Findings}

Incomplete or misleading information about sunscreen is common on YouTube and may play a role in sunscreen misuse, as reported in the literature [4,5]. In light of research on the quality of videos covering other areas of health information, the variable accuracy we found with respect to sunscreen use is not surprising [9]. Consistent with previous literature, no videos produced by government health agencies or cancer-related NGOs were among the most popular or relevant [13]. The EWG was the most-cited source of scientific information, but these video references were typically decontextualized statements about possible harms of common sunscreen chemicals (eg, oxybenzone and octinoxate) used in pitches to promote and sometimes sell other products. The outdated terms "sunblock" and "waterproof" were rarely used, but the videos generally failed to include all standard recommendations for effective sunscreen use or to recommend a combined sun protection approach. In the absence of instruction on application thickness, reapplication, and complementary sun protection measures, the emphasis we observed on SPF and broad-spectrum classification may perpetuate ineffective sunscreen use.

\section{Unexpected Findings}

Some videos contradicted sun safety recommendations, warned about health risks or dangerous ingredients of sunscreen, or included do-it-yourself recipes. Although these videos were not the majority, this finding is surprising and somewhat alarming in that some of these videos featured health care-affiliated spokespersons. These providers represented a subset of health care providers on YouTube and were primarily promoters of alternative or naturopathic medicine. The do-it-yourself sunscreen tutorial was a concerning and unexpected content category, as our search strategy did not target this content. Do-it-yourself sunscreen recipes have not undergone the testing that is required by the FDA and cannot be assumed to be effective. Some "recipes" included no approved sunscreen ingredients and instead promoted ingredients such as green tea and coconut oil. Clinicians should be aware of such videos and should be prepared to address the topic with patients who are considering making their own sunscreen. While recent research on sunscreen absorption [22] may raise public concern, especially about the use of sunscreen by children, the extant recommendations are unchanged; we can conclude from available evidence that sunscreen is safe and that its use is superior to unprotected exposure for children and adults (for infants under 6 months of age, sunscreen is not recommended; rather, sun exposure should be avoided completely) [23]. Guidance such as use of a mineral sunscreen, sun avoidance strategies, and use of protective clothing and hats [23] may be particularly useful to parents who are concerned about using sunscreen products on children.

\section{Limitations}

The YouTube videos analyzed here represent one point in time. Videos that are highly popular at one point may fade in popularity over time and may therefore not be prominent in subsequent searches. In our approach, we intentionally used neutral search terms associated with sunscreen and then gauged the valence of the most popular and relevant videos. Positive or negative search terms may yield different types of content; also, the search algorithms such as "relevance" used by YouTube are proprietary and may change over time, thus yielding different search results. Sunscreen videos are a narrow topic, and the most popular videos still do not qualify as generally popular, as viral videos have millions of views. Furthermore, not all adults use sunscreen, and individuals 
seeking information about sunscreen specifically from government health agencies may not use the YouTube application programming interface and may instead use Google or search the agency's website. The number of videos contradicting standard recommendations for sun safety or demonstrating do-it-yourself sunscreen was unanticipated; however, the behavioral significance of this finding is unclear.

\section{Conclusion}

Sunscreen use is a common and important form of sun protection, and YouTube is a common source of health information; it is especially popular among teens and young adults, which are the developmental groups with the highest rates of sunburn [3]. This study fills an important gap in the literature by offering a comprehensive examination of sunscreen information on YouTube, a common source of information for young people. The public currently bears the burden of judging the credibility of sunscreen messages; however, a challenge inherent in our findings is that health care providers depicted in videos (who are generally trusted sources of information) were not, as a rule, arbiters of high-quality sunscreen information. While the popular videos sampled were generally positive toward sunscreen use, the information content appears to be problematic in 2 ways. First, sunscreen-related content on YouTube infrequently recommends complementary sun safety strategies. This is particularly problematic when videos explicitly discourage sunscreen use [24]. Second, a small but not unimportant percentage of popular videos contained misleading information.

YouTube is a promising tool to share information on effective sunscreen use; however, incomplete or negative content about sunscreen is common, and in some cases, this content is communicated by health care-affiliated messengers. Further, health agencies that produce YouTube videos related to sun protection were not among the more popular or relevant video sources. These data highlight a challenge of using YouTube for public health communication: how to create eye-catching content that will reliably be identified by social media search algorithms. To better harness YouTube for disseminating sun safety messages, health agencies may look to increase their content visibility by better incorporating features such as memorable titles and keyword tags to increase their popularity. Considering the increasing popularity of YouTube as a source of health information, further research is warranted to examine viewer response to these videos and to determine whether exposure to the videos impacts subsequent sun safety behavior.

\section{Acknowledgments}

The authors would like to acknowledge the contributions of Anne Hartman, who assisted in codebook development.

\section{Conflicts of Interest}

None declared.

\section{References}

1. Guy GP, Machlin SR, Ekwueme DU, Yabroff KR. Prevalence and costs of skin cancer treatment in the U.S., 2002-2006 and 2007-2011. Am J Prev Med 2015 Feb;48(2):183-187 [FREE Full text] [doi: 10.1016/j.amepre.2014.08.036] [Medline: 25442229]

2. Armstrong BK, Kricker A. The epidemiology of UV induced skin cancer. J Photoch Photobio B 2001 Oct;63(1-3):8-18. [doi: 10.1016/s1011-1344(01)00198-1]

3. Holman DM, Ding H, Guy GP, Watson M, Hartman AM, Perna FM. Prevalence of Sun Protection Use and Sunburn and Association of Demographic and Behaviorial Characteristics With Sunburn Among US Adults. JAMA Dermatol 2018 May 01;154(5):561-568 [FREE Full text] [doi: 10.1001/jamadermatol.2018.0028] [Medline: 29541756]

4. Pratt H, Hassanin K, Troughton LD, Czanner G, Zheng Y, McCormick AG, et al. UV imaging reveals facial areas that are prone to skin cancer are disproportionately missed during sunscreen application. PLoS One 2017;12(10):e0185297 [FREE Full text] [doi: 10.1371/journal.pone.0185297] [Medline: 28968413]

5. US Dept of Health and Human Services, Office of the Surgeon General. 2014. The Surgeon General's Call to Action to Prevent Skin Cancer URL: https://www.hhs.gov/sites/default/files/call-to-action-prevent-skin-cancer.pdf [accessed 2020-04-16]

6. Morris KL, Perna FM. Decision Tree Model vs Traditional Measures to Identify Patterns of Sun-Protective Behaviors and Sun Sensitivity Associated With Sunburn. JAMA Dermatol 2018 Aug 01;154(8):897. [doi: 10.1001/jamadermatol.2018.1646]

7. 21CFR201.: U.S. Food and Drug Administration Code of Federal Regulations Title 21 URL: https://www.accessdata.fda.gov/ scripts/cdrh/cfdocs/cfCFR/CFRSearch.cfm?fr=201.327 [accessed 2019-10-25]

8. Petersen B, Wulf HC. Application of sunscreen--theory and reality. Photodermatol Photoimmunol Photomed 2014;30(2-3):96-101. [doi: 10.1111/phpp.12099] [Medline: 24313722]

9. Farberg AS, Glazer AM, Rigel AC, White R, Rigel DS. Dermatologists' Perceptions, Recommendations, and Use of Sunscreen. JAMA Dermatol 2017 Jan 01;153(1):99. [doi: 10.1001/jamadermatol.2016.3698]

10. Koch-Weser S, Bradshaw YS, Gualtieri L, Gallagher SS. The Internet as a health information source: findings from the 2007 Health Information National Trends Survey and implications for health communication. J Health Commun 2010 Dec;15 Suppl 3:279-293. [doi: 10.1080/10810730.2010.522700] [Medline: 21154099] 
11. Chou WS, Hunt YM, Beckjord EB, Moser RP, Hesse BW. Social media use in the United States: implications for health communication. J Med Internet Res 2009 Nov;11(4):e48 [FREE Full text] [doi: 10.2196/jmir.1249] [Medline: 19945947]

12. Perrin A. Social Media Usage: 2005-2015. 2005. Pew Research Center 2015 Oct 8 URL: https://www.pewinternet.org/ 2015/10/08/social-networking-usage-2005-2015/ [accessed 2019-10-25]

13. Mitchell SJ, Godoy L, Shabazz K, Horn IB. Internet and Mobile Technology Use Among Urban African American Parents: Survey Study of a Clinical Population. J Med Internet Res 2014 Jan 13;16(1):e9. [doi: 10.2196/jmir.2673]

14. Alexa. The top 500 sites on the web URL: https://www.alexa.com/topsites [accessed 2019-10-25]

15. Madathil KC, Rivera-Rodriguez AJ, Greenstein JS, Gramopadhye AK. Healthcare information on YouTube: A systematic review. Health Informatics J 2015 Sep;21(3):173-194. [doi: 10.1177/1460458213512220] [Medline: 24670899]

16. Keelan J, Pavri-Garcia V, Tomlinson G, Wilson K. YouTube as a source of information on immunization: a content analysis. JAMA 2007 Dec 5;298(21):2482-2484. [doi: 10.1001/jama.298.21.2482] [Medline: 18056901]

17. Basch CH, Basch CE, Hillyer GC, Reeves R. YouTube Videos Related to Skin Cancer: A Missed Opportunity for Cancer Prevention and Control. JMIR Cancer 2015 Mar 02;1(1):e1. [doi: 10.2196/cancer.4204]

18. Ruppert L, Køster B, Siegert A, Cop C, Boyers L, Karimkhani C, et al. YouTube as a source of health information: Analysis of sun protection and skin cancer prevention related issues. Dermatol Online J 2017 Jan 15;23(1) [FREE Full text] [Medline: 28329465]

19. Sampson M, Cumber J, Li C, Pound CM, Fuller A, Harrison D. A systematic review of methods for studying consumer health YouTube videos, with implications for systematic reviews. PeerJ 2013 Sep;1:e147 [FREE Full text] [doi: 10.7717/peerj.147] [Medline: 24058879]

20. Does Video Length Matter?. URL: https://wistia.com/learn/marketing/does-length-matter-it-does-for-video-2k12-edition [accessed 2020-04-16]

21. StataCorp. Stata Statistical Software: Release 14. College Station, TX: Statacorp LP; 2015. URL: https://www.stata.com/ stata14/ [accessed 2020-04-16]

22. Matta MK, Zusterzeel R, Pilli NR, Patel V, Volpe DA, Florian J, et al. Effect of Sunscreen Application Under Maximal Use Conditions on Plasma Concentration of Sunscreen Active Ingredients. JAMA 2019 Jun 04;321(21):2082. [doi: 10.1001/jama.2019.5586]

23. Holman DM, Qin J, Gottschlich EA, Balk SJ. Clinical counseling on sun protection and indoor tanning avoidance: A survey of current practices among U.S. health care providers. Prev Med 2019 Sep;126:105783. [doi: 10.1016/j.ypmed.2019.105783]

24. Bleakley A, Lazovich D, B Jordan A, Glanz K. Compensation Behaviors and Skin Cancer Prevention. Am J Prev Med 2018 Dec;55(6):848-855. [doi: 10.1016/j.amepre.2018.06.019] [Medline: 30344038]

\author{
Abbreviations \\ CDC: Centers for Disease Control and Prevention \\ EWG: Environmental Working Group \\ FDA: Food and Drug Administration \\ SPF: sun protection factor \\ UV: ultraviolet
}

Edited by G Eysenbach; submitted 18.04.19; peer-reviewed by C Basch, E Warner, K Glanz; comments to author 01.10.19; revised
version received 20.11.19; accepted 22.03.20; published 02.06.20
Please cite as:
Julian AK, Welch J, Bean MM, Shahid S, Perna FM
Information About Sunscreen on YouTube and Considerations for Sun Safety Promotion: Content Analysis
JMIR Dermatol 2020;3(1):e14411
URL: http://derma.jmir.org/2020/1/e14411/
doi: $\underline{10.2196 / 14411}$
PMID:

(C)Anne K Julian, Jessica Welch, Maddison M Bean, Sarah Shahid, Frank M Perna. Originally published in JMIR Dermatology (http://derma.jmir.org), 02.06.2020. This is an open-access article distributed under the terms of the Creative Commons Attribution License (https://creativecommons.org/licenses/by/4.0/), which permits unrestricted use, distribution, and reproduction in any medium, provided the original work, first published in JMIR Dermatology Research, is properly cited. The complete bibliographic information, a link to the original publication on http://derma.jmir.org, as well as this copyright and license information must be included. 